\title{
COMPORTAMENTO DO ARMAZENAMENTO DE ÁGUA ENTRE OS ANOS DE 2002 A 2015 PARA A REGIÃO HIDROGRÁFICA BRASILEIRA DO ATLÂNTICO NORDESTE ORIENTAL CONSIDERANDO OBSERVAÇÕES GRACE, TRMM E IN SITU
}

\author{
WATER STORAGE BEHAVIOUR BETWEEN THE YEARS 2002 TO 2015 FOR THE BRAZILIAN \\ ORIENTAL NORTHEAST ATLANTIC HYDROGRAPHIC REGION CONSIDERING GRACE, \\ TRMM AND IN SITU OBSERVATIONS
}

\section{Tarso Luconi ROSENHAIM, Rodrigo Mikosz GONÇALVES, Josilene Pereira LIMA, Nicolas Alexandre GAMA}

\begin{abstract}
Universidade Federal de Pernambuco (UFPE), Centro de Tecnologia e Geociências, Departamento de Engenharia Cartográfica, Programa de Pós-Graduação em Ciências Geodésicas e Tecnologias da Geoinformação. Recife-PE.
\end{abstract}

Emails: tarso78@hotmail.com; rodrigo.mikosz@ufpe.br; josilenelima2003@yahoo.com.br; nicolasgama_@hotmail.com

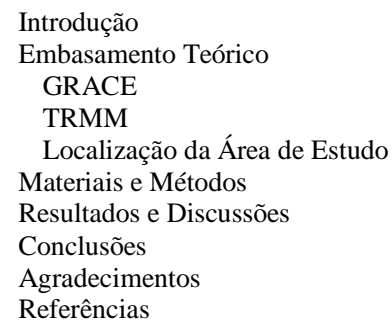

\begin{abstract}
RESUMO - Um dos problemas para gestão de bacias hidrográficas é a obtenção de informações temporais capazes de abranger toda a bacia. Nesta contribuição destacam-se o emprego das observações procedentes da missão GRACE (Gravity Recovery And Climate Experiment), TRMM (Tropical Rainfall Measuring Mission), estações pluviométricas e níveis de reservatórios in situ, aplicadas na avaliação das reservas de água contida na Região Hidrográfica Brasileira do Atlântico Nordeste Oriental. Os objetivos são: estudar o comportamento das variações temporais de armazenamento de água na bacia; estimar o volume de água equivalente para dois instantes sazonais (fim do $1^{\circ}$ e do $2^{\circ}$ semestre) para os anos de 2002 e 2015; e correlacionar os dados provenientes dos satélites artificiais com amostras de estações em campo (pluviométricas e nível de reservatórios). Os resultados apresentam decréscimos de aproximadamente $23.710 \mathrm{hm}^{3}$ para o final do primeiro semestre e de $19.246 \mathrm{hm}^{3}$ para o final do segundo semestre nas comparações de 2002 com 2015 , bem como a importância do monitoramento hidrográfico utilizando informações de satélites artificiais. Neste estudo se destaca que a bacia a partir do ano de 2012 até 2015 apresentou declínio em seu armazenamento de água causando escassez no abastecimento para a sociedade.
\end{abstract}

Palavras-chave: GRACE, gravimetria, geodésia ambiental, recursos hídricos.

\begin{abstract}
One of the problems concerning watershed management is about obtaining temporal information capable of covering the entire basin. In this contribution, it was highlighted the use of observations from GRACE (Gravity Recovery And Climate Experiment), TRMM (Tropical Rainfall Measuring Mission), rainfall stations and in situ reservoirs levels, applied to the evaluation of water supply in the Brazilian Hydrographic Region of the Oriental Northeast Atlantic. The goals are: study the behavior of temporal variations of water storage in the basin; estimate the volume of water equivalent for two seasonal moments (end of $1^{\text {st }}$ and $2^{\text {nd }}$ semester) for the years 2002 and 2015; and correlate data from artificial satellites with samples from field stations (rainfall and reservoir level). The results showed decreases nearly $23,710 \mathrm{hm}^{3}$ for the end of the first semester and $19,246 \mathrm{hm}^{3}$ for the end of the second semester comparing 2002 with 2015, as well as the importance of hydrographic monitoring using artificial satellites. This study highlights that the basin from the year 2012 until 2015 presented water storage decline causing supply scarcity to the society.
\end{abstract}

Keywords: GRACE, gravimetry, environmental geodesy, water resources.

\section{INTRODUÇÃO}

A Região Hidrográfica Atlântico Nordeste Oriental (RHANO), escolhida como estudo de caso, localiza-se no extremo nordeste brasileiro, desde o Piauí até Alagoas, tem cerca de 286 mil $\mathrm{km}^{2}$ de área, onde vivem aproximadamente 24,1 milhões de pessoas, abrangendo boa parte do semiárido brasileiro.

O conceito de bacia hidrográfica refere-se a uma área de captação natural da água de precipitação que faz convergir o escoamento para um único ponto de saída. A bacia hidrográfica compõe-se de um conjunto de superfícies vertentes e de uma rede de drenagem formada por cursos de água que confluem até resultar em um leito único no seu exutório (Tucci, 2007).

Entender o comportamento temporal a respeito dos recursos hídricos armazenados nas regiões hidrográficas brasileiras, bem como verificar tendências ao longo do tempo são importantes informações para a eficiência na gestão desse bem natural, que afeta diretamente 
o provimento à população, agricultura e indústrias.

Atualmente, as observações temporais por satélites artificiais fazem parte de uma gama de ferramentas investigatórias disponíveis para auxiliar no estudo e monitoramento dos recursos hídricos, e vem sendo aplicadas em diversas regiões do planeta, tais como os estudos de caso apresentados por: Awange et al. (2013) e Ndehedehe et al. (2016) na Africa, Chen et al. (2016) na Austrália, Molodtsova et al. (2016) nos EUA, entre outros.

Dentre outras aplicações utilizando satélites artificiais destacam-se aqui os mapas gravimétricos da Terra provenientes da missão GRACE (Gravity Recovery And Climate Experiment), capazes de fornecerem informações sobre o comportamento do armazenamento de água total (em inglês TWS Total Water Storage), sendo compostos pelo conjunto de águas superficiais, subterrâneas e umidade do solo, conforme demonstrados em alguns trabalhos aplicados no Brasil entre eles: Getirana (2016), no qual é apresentado uma aplicação fazendo uso dos dados da missão GRACE para detectar e quantificar a prolongada seca que afeta o sudeste do Brasil desde 2012 e fornecer estimativas de áreas impactadas e déficits de água específicas da região; Sun et al. (2016) fazem uso de dados dos satélites GRACE para avaliar e quantificar a seca em curso sobre a Bacia Hidrográfica do Rio São Francisco de 2012 a 2015; e Xavier et al. (2013), destacam um panorama sobre a possibilidade de utilização do GRACE em modelagens hidrológicas de grandes bacias, com enfoque na região Amazônica, demonstrando a importância destas observações temporais para a estimativa do comportamento sazonal e interanual do armazenamento total de água.

Salientando a importância da obtenção e processamento de informações sobre os recursos hídricos voltados ao planejamento estratégico, Gutiérrez et al. (2014) assinalam que as secas ocorridas no nordeste e sul brasileiro nos últimos anos ocasionaram o surgimento de rodadas de discussões para melhorar a política de gestão a níveis federais e estaduais baseados em um estudo de caso para o Brasil feito pelo Banco Mundial dando indicativos sobre medidas e abordagens para o problema da seca.

Na América do Sul, Montecino et al. (2016) destacaram outra aplicabilidade do GRACE com enfoque para águas subterrâneas na região norte do Chile. No referido estudo foi feito o monitoramento destas águas utilizando as soluções mensais da missão GRACE correlacionadas com observações de poços fornecidos pela Direção Geral dos Recursos Hídricos (DGA) do Ministério das Obras Públicas daquele país.

Outras informações provenientes de satélites artificiais podem ser um complemento para análises hidrográficas temporais como é o caso da precipitação fornecida pelo TRMM (Tropical Rainfall Measuring Mission) através do Rainfall Product (3B43). Além disso, observações amostrais in situ disponíveis nas estações pluviométricas do INMET (Instituto Nacional de Meteorologia) e ANA (Agência Nacional de Águas) tornam-se um complemento fundamental para validar os produtos obtidos por satélites.

Diante do exposto, fazendo uso de diferentes fontes de observações para detectar mudanças na Região Hidrográfica Brasileira do Atlântico Nordeste Oriental, o presente estudo têm como objetivos: (i) estudar o comportamento da precipitação na bacia em questão utilizando dados provenientes do TRMM; (ii) validar a correlação dos dados do TRMM com observações em campo; (iii) analisar o comportamento temporal provenientes da capacidade de água armazenada de nos principais reservatórios considerando a bacia em estudo (iv) utilizar o GRACE para verificar o comportamento temporal entre abril de 2002 até dezembro de 2015, intervalo este considerando o início de operação desta missão até o início do presente estudo; (v) a partir das observações obtidas pelo GRACE selecionar os meses que representam o final do regime de maiores e menores precipitações na área de estudo sendo: maio de 2002, novembro de 2002, abril de 2015 e dezembro de 2015, para calcular numericamente o volume de água equivalente e verificar assim a mudanças de comportamento considerando um intervalo de 13 anos. 


\section{EMBASAMENTO TEÓRICO}

O estudo dos temas considerados relevantes para o desenvolvimento do presente trabalho foram: as missões GRACE e TRMM, dados oriundos de coletas de estações meteorológicas, bem como a descrição das regiões hidrográficas brasileiras, em particular a Região Hidrográfica do Atlântico Nordeste Oriental.

\section{GRACE}

O GRACE é resultado de uma parceria entre as agências espaciais Estadunidense - NASA (National Aeronautics and Space Administration) e da Alemanha - DLR (Deutsche Forschun gsanstalt für Luft und Raumfahrt), que tem como objetivo a obtenção de medidas precisas do campo gravimétrico terrestre (Tapley \& Reigber, 2001). A missão GRACE consiste em dois satélites artificiais idênticos na mesma órbita, cerca de 220 $\mathrm{km}$ um do outro e $500 \mathrm{~km}$ sobre a Terra. A massa de cada um dos satélites é de $487 \mathrm{~kg}$ e sua fonte de energia é solar. A primeira missão foi lançada em março de 2002 com vida útil prevista de 5 anos, entretanto foi estendida e ainda está ativa considerando o ano de 2016 (Watkins \& Bettadpur, 2000).

Apesar de pequenas variações nas especificações técnicas, que são monitoradas constantemente, em geral a inclinação da órbita relativa ao plano equatorial (i) dos satélites é quase polar, com excentricidade orbital $(e)$ elíptica. Possui período orbital $(T)$ de 91 minutos, ou seja, circunda a Terra quase 16 vezes por dia, registrando variações de minutos do campo gravitacional terrestre.

O GRACE não utiliza para o monitoramento global o desenvolvimento da obtenção de imagens da superfície terrestre por meio da detecção e medição quantitativa das respostas das interações da radiação eletromagnética com os materiais terrestres, preceito básico do sensoriamento remoto (Meneses \& Almeida, 2012). Em vez disso, a missão usa um sistema da variação microondas para medir com precisão as alterações na velocidade e distância entre as duas espaçonaves "gêmeas". A intercomunicação entre os dois satélites é efetuada através de uma ligação na banda $K$ no espectro das micro-ondas, permitindo medir distâncias e variações de distâncias entre os dois satélites com uma precisão de aproximadamente 10 micrômetros.

Quando o primeiro satélite passa por uma região com gravidade ligeiramente mais forte, isto é, uma anomalia gravitacional, esta o impele (empuxo) levemente mais para a frente do satélite que o segue. Causando, assim, um aumento na distância entre os dois satélites. Então o primeiro satélite, após passar a anomalia, retarda sua velocidade, enquanto que o outro acelera e reduz no mesmo ponto da alteração gravitacional. Medindo a constante variação de distância entre os dois satélites e combinando com dados precisos de instrumentos de sistemas globais de navegação por satélites (GNSS), são feitos os mapas detalhados das anomalias gravitacionais da Terra.

Para se ter uma boa solução para o campo de gravidade é necessário a acumulação de dados de rastreamento dos satélites da missão por cerca de um mês. Sendo assim os mapas do campo de gravidade terrestre formados a partir do GRACE são mensais, mostrando variações no campo gravitacional não só de um local para outro, mas também temporalmente (Tapley et al., 2004).

As mudanças mensais na gravidade são causadas por alterações mensais de massa na superfície terrestre e sobre ela (Wahr et al., 1998). Em um perfil vertical onde a espessura da camada de água pode ter dimensões quilométricas as variações de massa mensais perto da superfície terrestre são pequenas, na ordem de centímetros. Então os dados GRACE são apresentados como "espessura de água equivalente" (water equivalent tchickness), com unidade em centímetros, sendo conhecidos também como "centímetros de água equivalente". Os produtos são apresentados na forma de malha (grid) e utilizados para calcular as mudanças de massa em termos de superfície de altura equivalente de água, mais informações podem ser encontradas em Wahr et al., (1998).

\section{TRMM}

O Satélite Tropical Rainfall Measuring Mission - TRMM, é resultado de um projeto desenvolvido em uma parceria entre Estados Unidos da América - EUA e Japão. Foi lançado a bordo do veículo espacial H-II F6 em 28 de novembro de 1997, no Centro Espacial de Tanegashima. O Japão ficou responsável pelo lançamento, e por desenvolver o instrumento de observação Precipitation Radar $\mathrm{PR}$, enquanto que os EUA por intermédio da National Aeronautics and Space Administration NASA, ficou responsável pela construção do veículo de lançamento, pelo desenvolvimento de 
outros quatro instrumentos de observação (TRMM Microwaver Imager - TMI, Visible and Infrared Scanner - VIRS, Cloud and Earth Radiant Energy Sensor - CERES, Lightning Imaging Sensor LIS), e pelo sistema de operação do satélite (Jaxa, 2015; Kummerow et al., 1998).

Os dados com as estimativas de precipitação utilizados no presente estudo provêm do produto 3B43 v7, sendo disponibilizado em formato Hierarchical Data Format (.HDF) com o tamanho do pixel de $0.25^{\circ} \times 0.25^{\circ}$ e abrangendo de $50^{\circ} \mathrm{N}$ até $50^{\circ} \mathrm{S}$ de Latitude. Os dados de precipitação remetem a uma série temporal média mensal de chuva em milímetros por hora $\left(\mathrm{mm} \mathrm{hr}^{-1}\right)$.

Este produto do TRMM é um algoritmo que combina dados de múltiplos sensores orbitais originando, desta forma, os dados de precipitação global (Huffman et al., 1995, 2007). De um modo geral, como vantagem o lançamento do satélite TRMM permitiu a obtenção de informações sobre as características da precipitação em lugares que possuem uma baixa densidade de redes de estações meteorológicas.

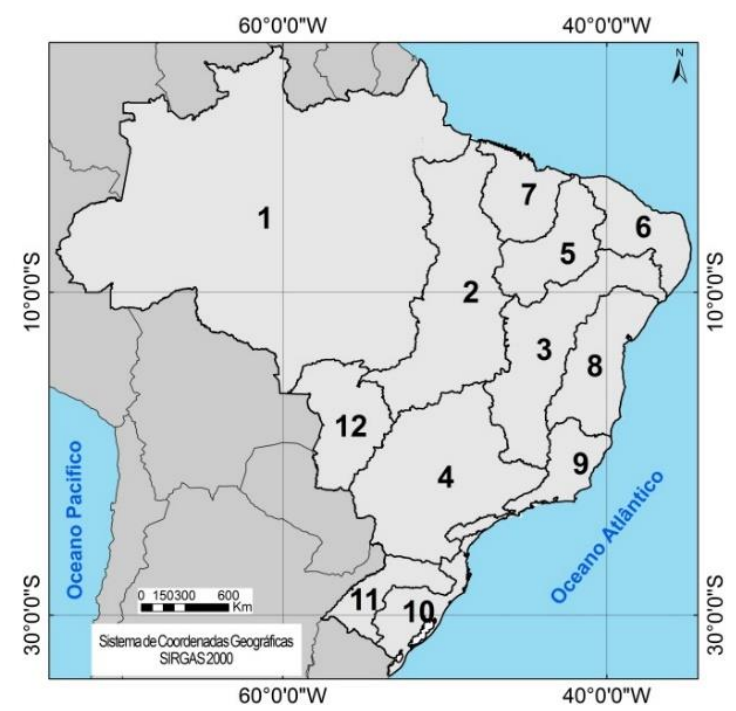

Figura 1 - Regiões Hidrográficas Brasileiras (CNRH, 2003 - Resolução nº 32).

O regime climático da Região Hidrográfica do Atlântico Nordeste Oriental é caracterizado por um período com maiores precipitações, em geral o primeiro semestre do ano, e com pouca

\section{Localização da Área de Estudo}

A Região Hidrográfica Brasileira foi determinada como o espaço territorial brasileiro compreendido por uma bacia, grupo de bacias ou sub-bacias hidrográficas contíguas com características naturais, sociais e econômicas homogêneas ou similares, com vistas a orientar o planejamento e gerenciamento dos recursos hídricos (CNRH, 2003 - Resolução n ${ }^{\circ}$ 32). As 12 regiões hidrográficas brasileiras são: (1) Amazônica, (2) Tocantins-Araguaia, (3) São Francisco, (4) Paraná, (5) Parnaíba, (6) Atlântico Nordeste Oriental, (7) Atlântico Nordeste Ocidental, (8) Atlântico Leste, (9) Sudeste, (10) Atlântico Sul, (11) Uruguai e (12) Paraguai (Figura 1).

A Região Hidrográfica do Atlântico Nordeste Oriental, foco de estudo do presente trabalho, abrange uma pequena parte do litoral do estado do Piauí, quase a totalidade do Ceará, todo o Rio Grande do Norte e Paraíba, a porção litorânea de Pernambuco e parte da de Alagoas (Figura 2). É limitada a sul pela região hidrográfica do São Francisco e a oeste pela do Parnaíba.

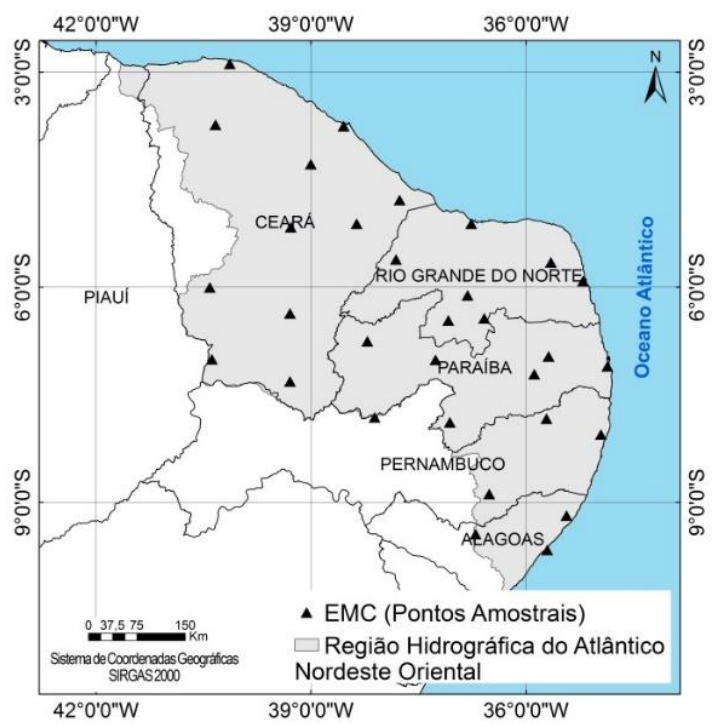

Figura 2 - Polígono delimitando a Região Hidrográfica do Atlântico Nordeste Oriental, onde os triângulos representam as estações meteorológicas utilizadas no estudo.

ou nenhuma precipitação, no segundo semestre. Mais informações a respeito das bacias hidrográficas brasileiras além desta em estudo podem ser encontradas em ANA (2009) e ANA (2015).

\section{MATERIAIS E MÉTODOS}

Os dados da missão TRMM são disponibilizados gratuitamente via página do PMM - Precipitation Measurement Missions (https://pmm.nasa.gov/data-access/downloads/ trmm). Os dados foram obtidos em formato NETCDF, estes foram inseridos num ambiente GIS (Geographic Information System), utilizando o software ArcGIS desenvolvido pela 
ESRI, onde foram extraídas as informações necessárias para o presente estudo.

Os dados das Estações Meteorológicas Convencionais - EMC, foram obtidos junto ao Banco de Dados Meteorológicos para Ensino e Pesquisa - BDMEP, do Instituto Nacional de Meteorologia - INMET, estes dados são fornecidos gratuitamente via site próprio (http://www.inmet.gov.br/portal/index.php?r=bd mep/bdmep), através de uma pesquisa utilizando filtros foi possível selecionar informações contendo a latitude, longitude e precipitação mensal de amostras pontuais na área de estudo.

Os dados da missão GRACE (Swenson, 2012, Landerer \& Swenson, 2012, Swenson \& Warh, 2006) são disponibilizados gratuitamente via página própria (http://grace.jpl.nasa.gov/), vinculada ao JLP (Jet Propulsion Laboratory), centro tecnológico responsável pelo desenvolvimento e controle dos satélites não tripulados da NASA, com suporte do programa MEaSURESs. Para este caso foram utilizadas informações já registradas espacialmente no formato GEOTIFF.

Para complementar o estudo e as análises sobre a bacia, foram adquiridas as observações a respeito da disponibilidade de água nos principais reservatórios da Região Hidrográfica do Atlântico Nordeste Oriental. As estações utilizadas foram obtidas através do site da Agência Nacional de Águas (ANA) e estão representadas no mapa da figura 3 .

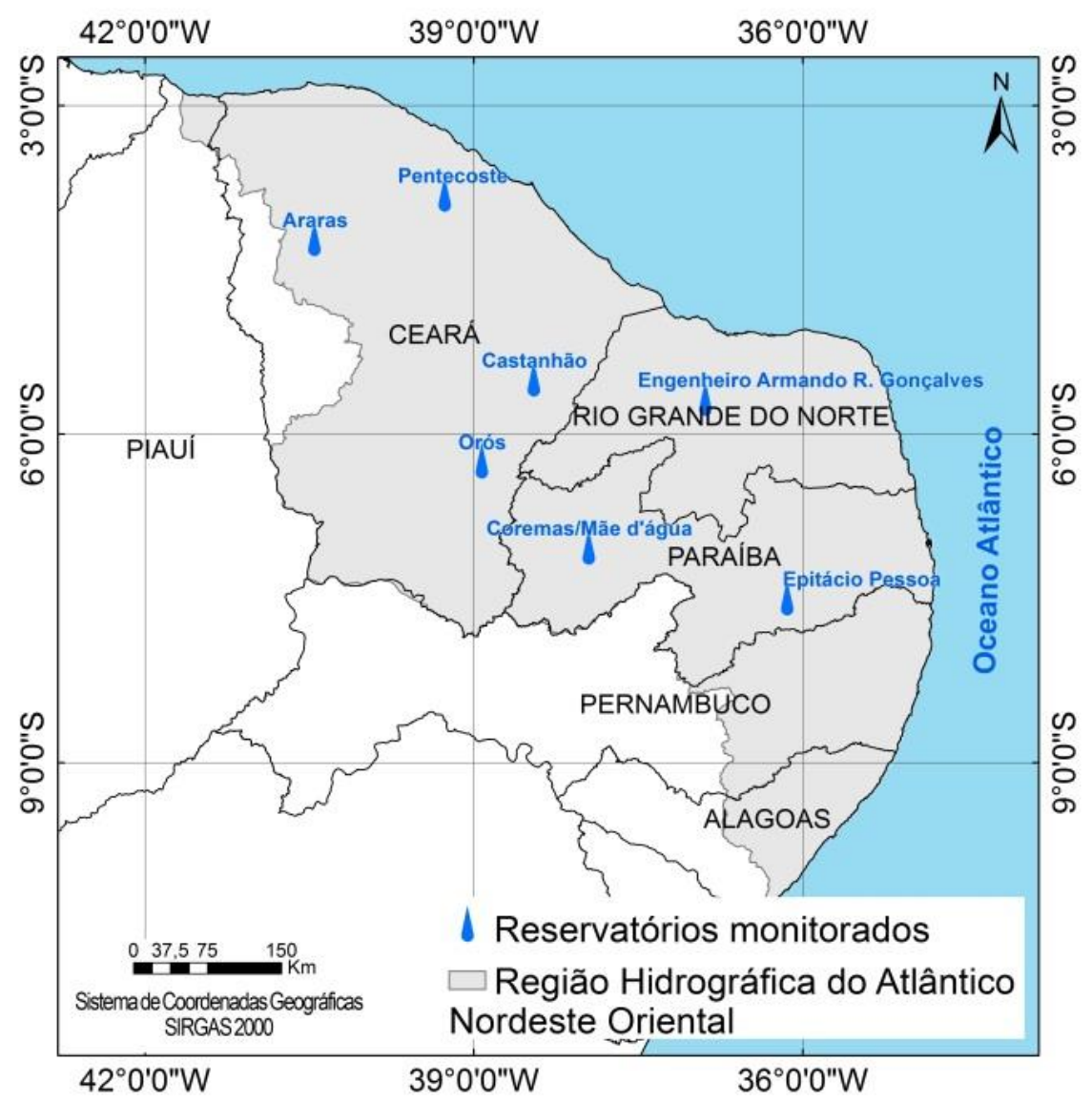

Figura 3 - Localização dos reservatórios utilizados no estudo.

Com o intuito de analisar as variações de espessura de água equivalente para Região Hidrográfica do Atlântico Nordeste Oriental de 2002 a 2015 (objetivos iv e $v$ ), e calcular a diferença entre os extremos, foram utilizadas as observações dos seguintes meses: maio, abril, novembro e dezembro. Essa seleção representa, respectivamente, a parte sazonal final dos regimes de maiores e de menores precipitações na região.
Na etapa de processamento dos dados foi feita uma reclassificação do grid inicial para uma melhor visualização, incluindo a classificação da área representada por água oceânica destacada com uma cor diferente das demais.

Para quantificar os valores de espessura equivalente de água para a região de estudo nos meses selecionados e calcular a diferença entre os extremos fez-se necessário selecionar somente os pixels (ou células) que são equivalentes a área 
alvo. O arquivo com a delimitação da Região Hidrográfica do Atlântico Nordeste Oriental foi obtido da biblioteca virtual da Agência Nacional de Águas (ANA), deste coletou-se, também, o valor referente a área do polígono em questão, sendo igual a $285.702,7 \mathrm{Km}^{2}$. A diferenciação do mesmo mês, mas de anos diferentes, foi feita no software de GIS de duas formas: o primeiro utilizando a ferramenta de álgebra de mapas que faz a subtração da imagem matricial (raster) de uma época (abril e dezembro de 2015) pela da outra (maio e novembro de 2002) e o segundo modo quantificando as variações de espessura equivalente de água.

$\mathrm{Na}$ sequência, foram efetuados os cálculos estatísticos de espessura equivalente de água para o intervalo proposto bem como a diferença dos meses considerando os anos extremos (2015-2002). Com isso é possível obter valores de perda ou ganho de massa média por pixel. Onde a mudança no volume equivalente de água é obtida através do valor massa média por pixel multiplicado pela área.

Como complemento e para dar melhor suporte às análises dos resultados, foram estimadas as precipitações obtidas pelo satélite TRMM bem como a validação dos resultados do satélite com as amostras de informações em campo conforme as estações demonstradas no mapa da figura 2 .

Para a seleção dos reservatórios, foram considerados aqueles com maior capacidade de armazenamento de água presentes na área de estudo. A série temporal do volume armazenado foi coletada mensalmente e o cálculo do volume acumulado (\%) se refere ao volume acumulado dividido pela capacidade de armazenamento.

\section{RESULTADOS E DISCUSSÕES}

Comparação entre as precipitações obtidas com o TRMM e as estações meteorológicas.

As figuras 4 e 5 apresentam os resultados obtidos pela interpolação dos dados de precipitação das estações meteorológicas convencionais representando a distribuição das chuvas na região hidrográfica considerando os meses de junho e dezembro para os anos de 2002 e 2015. Ao mesmo tempo apresenta-se a estimativa de precipitação fornecida pelo produto mensal 3B43 v7 do TRMM, que contém a variação e distribuição de precipitação na região conforme a série temporal em estudo.

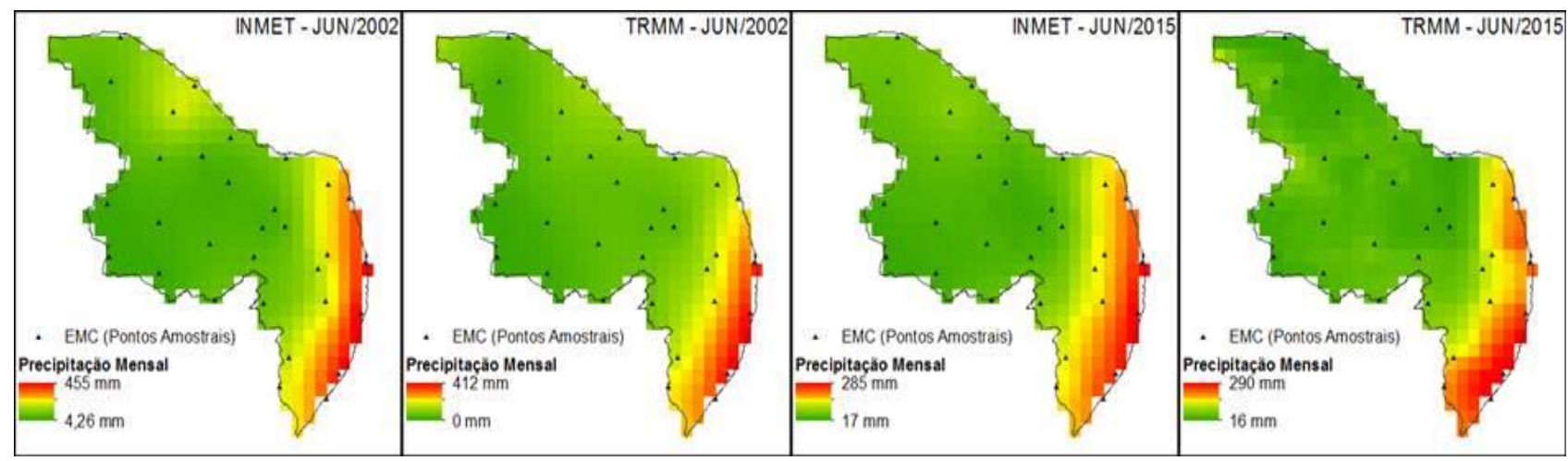

Figura 4 - Distribuição da precipitação sobre a Região Hidrográfica do Atlântico Nordeste Oriental no mês de junho de 2002 e 2015, observada pelas estações meteorológicas convencionais e o produto 3B43 v7 do TRMM.

Observa-se na figura 4 uma equivalência entre os dados de precipitação das estações meteorológicas convencionais e a estimativa de precipitação fornecida pelo TRMM, tanto para o mês de junho de 2002 quanto para junho de 2015. Esta equivalência é demostrada com uma maior incidência de precipitação, acima de 400 $\mathrm{mm}$ em junho de 2002 e perto de $300 \mathrm{~mm}$ em junho de 2015, na região litorânea que vai do litoral leste do estado do Rio Grande do Norte até Alagoas, e pouca precipitação no restante da área apresentada, tendo como valores abaixo de $5 \mathrm{~mm}$ para junho de 2002 e abaixo de $20 \mathrm{~mm}$ para junho de 2015. A figura 5, apresenta os resultados considerando o mês de dezembro (2002 e 2015), a equivalência entre os dois métodos não é tão simétrica, destacando a representatividade de maiores diferenças considerando o sudoeste e sul da área para dezembro de 2002 e sudeste para dezembro de 2015, e menores precipitações referem-se às mesmas partes da RHANO. 


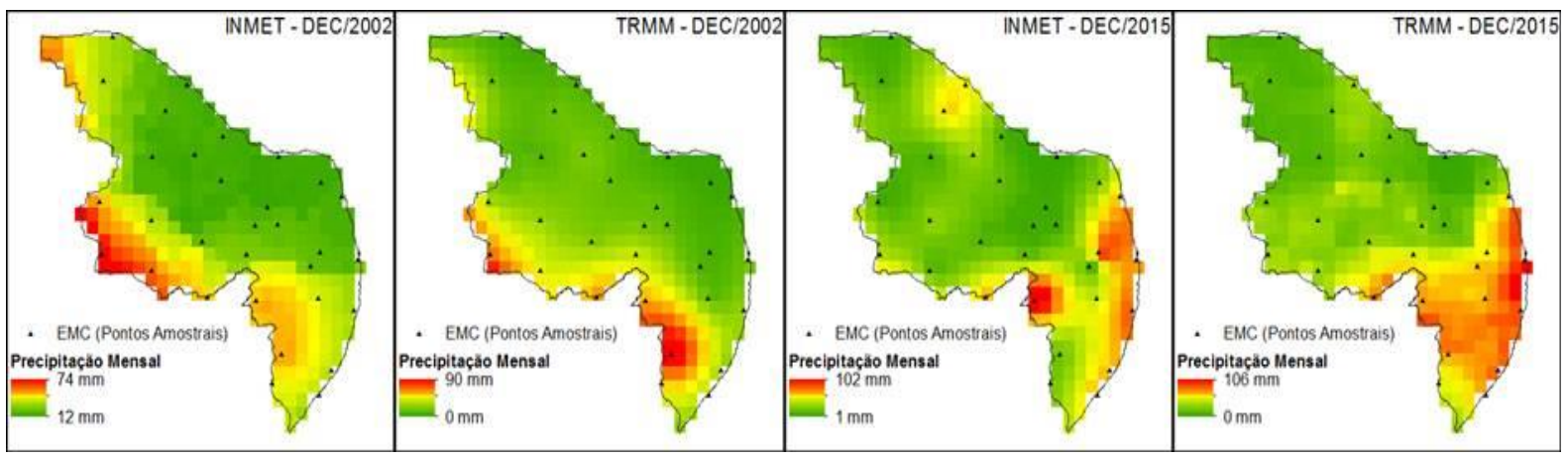

Figura 5 - Distribuição da precipitação sobre a Região Hidrográfica do Atlântico Nordeste Oriental no mês de dezembro de 2002 e 2015, observada pelas estações meteorológicas convencionais e o produto 3B43 v7 do TRMM.

A figura 6 apresenta a comparação do comportamento das duas séries temporais obtidas pelas estações meteorológicas convencionais e TRMM. Para este caso é possível destacar um comportamento semelhante conforme o que foi demonstrado nas figuras 4 e 5 , servindo desta forma como validação para a utilização da estimativa da precipitação fornecida pelo TRMM, este com a vantagem de abranger toda a bacia, ao invés de apenas dados pontuais obtidos pelas estações meteorológicas convencionais.

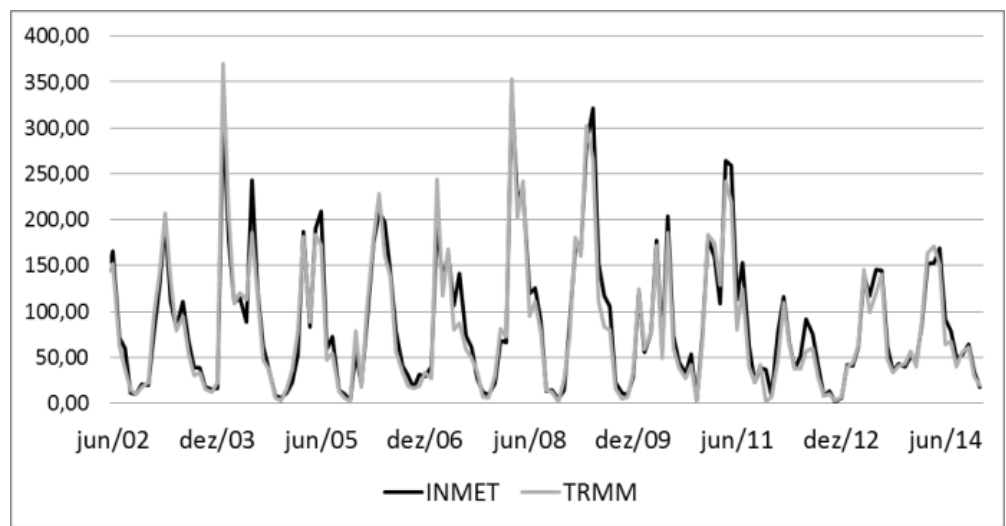

Figura 6 - Séries temporais, variação da precipitação na Região Hidrográfica Atlântico Nordeste Oriental.

Nas figuras 7 e 8 são apresentados os mapas com as diferenças de precipitação, sendo na figura 7 a diferença de precipitação observada pelos dois métodos em um mesmo período e na figura 8 a diferença de precipitação observadas pelo mesmo método para os meses de junho de 2002 e 2015 , e para os meses de dezembro de 2002 e 2015.
A figura 7 apresenta a comparação das duas fontes, representando as regiões com variações negativas e positivas, ou seja, uma forma de destacar as localidades onde as observações, quando comparadas, apresentam decréscimo ou acréscimo no valor de precipitação. Sendo assim, mais um recurso para verificar diferenças entre observações.

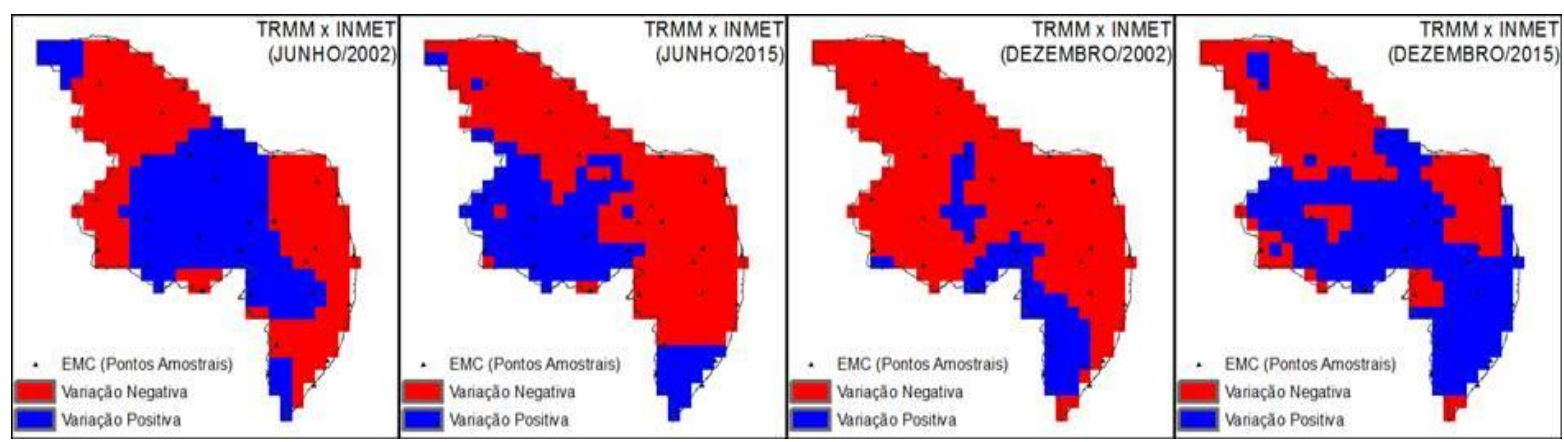

Figura 7 - Diferença de precipitação entre os métodos para os períodos de junho de 2002 e 2015 e dezembro de 2002 e 2015. 


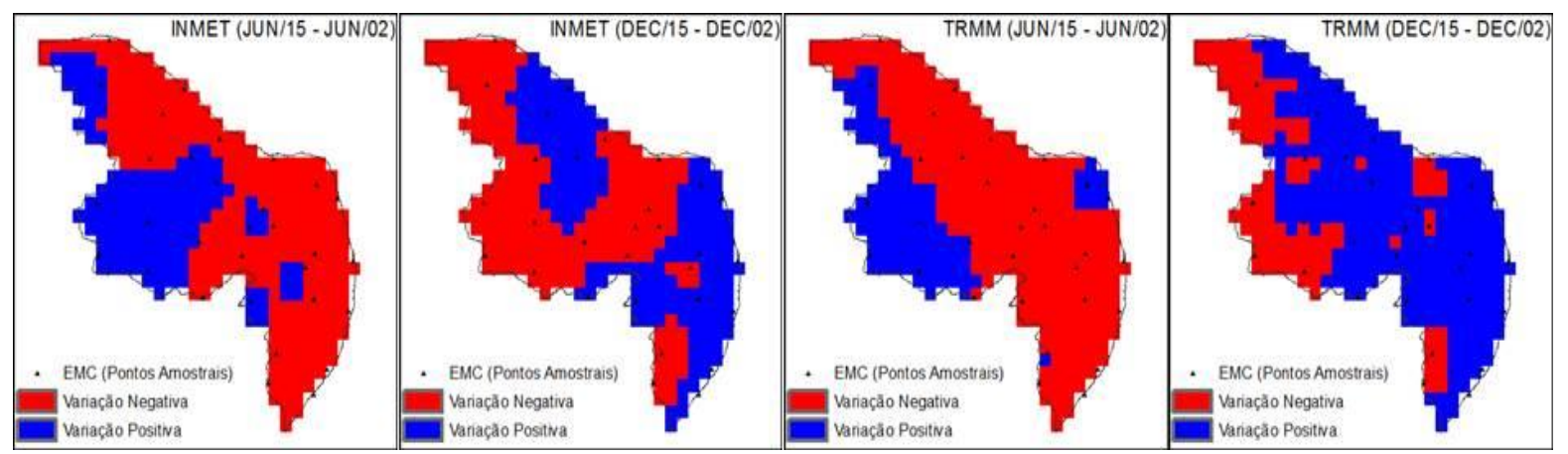

Figura 8 - Diferença de precipitação entre os períodos estudados.

A figura 8 apresenta os resultados da comparação considerando duas épocas sazonais de 2015 com relação às mesmas de 2002 para cada fonte de observações de precipitação destacando as regiões onde houve variações tanto negativas, como positivas.

O monitoramento da precipitação é de grande valia e pode auxiliar na análise e entendimento do comportamento das massas de água no continente e bacia em questão. A comparação efetuada nas figuras 4, 5, 6, 7 e 8 visaram atender os objetivos (i), (ii) e (iii) deste estudo apresentando a comparação entre dois métodos de observações deste fenômeno, e assim validar os dados provenientes das observações feitas pelo método orbital (TRMM), que apresentou uma forte correlação com os dados in situ, auxiliando, desta forma, para entender o comportamento temporal estudado.
Série temporal (2002 a 2015) entre as espessuras equivalente de água obtida pelo GRACE na Região do Atlântico Nordeste Oriental considerando o comportamento para os meses de maio e novembro.

Em 2002, quando os satélites da missão GRACE começaram a fornecer observações sobre o campo gravimétrico terrestre, os equipamentos ainda estavam passando por ajustes ocasionando meses sem dados. Somando-se a isso existem meses em que as órbitas dos satélites possuem um padrão de quase repetição ou sobreposição de outra órbita já percorrida e isto causa erros nos coeficientes dos esféricos harmônicos maiores e consequentemente na geração dos produtos disponíveis. Além disso, outra condição que ocasiona meses faltantes nas observações desde 2011 é o envelhecimento das baterias que precisam ser reajustadas com mais frequência.
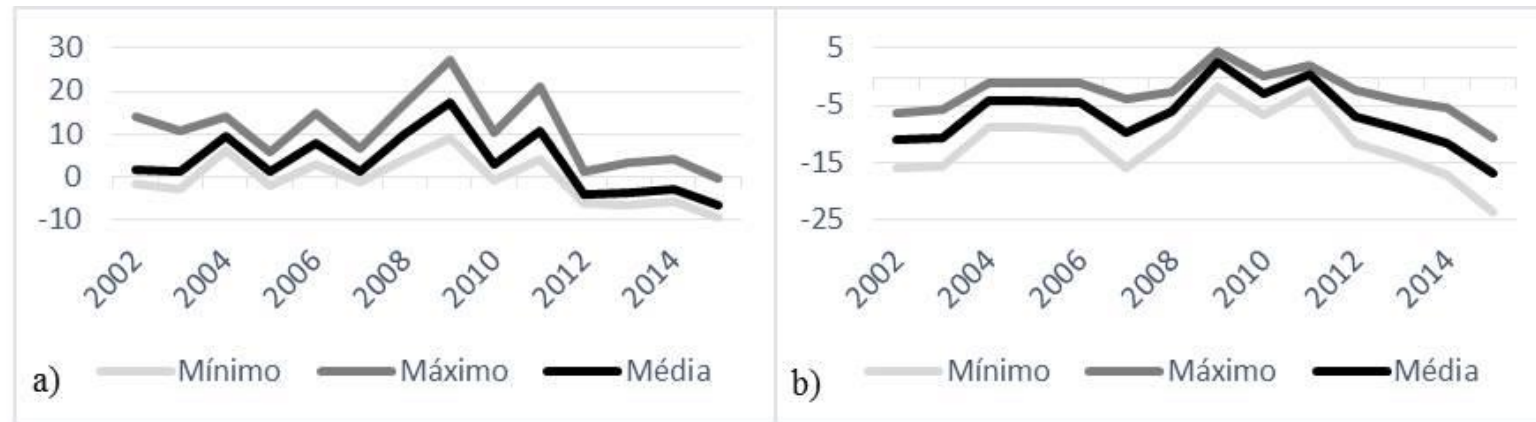

Figura 9 - Espessura equivalente de água (em cm) na Região do Atlântico Nordeste Oriental de 2002 a 2015: a) maio; b) novembro.

A figura 9 apresenta dois gráficos (a) e (b) com os valores de espessura equivalente de água considerando o mês de maio em (a) e em (b) novembro, ambos para o período compreendido de 2002 a 2015 na área estudada. Pelos motivos explanados anteriormente, maio de 2012 e 2015 são meses sem informações processadas para a serie temporal e para este caso especifico optou-se por preencher os mesmos com os respectivos valores dos meses mais próximos, e para o caso de novembro de 2015, que não possuía informações, foi substituído por dezembro. Os valores mínimo e máximo apesentados nos gráficos referem-se ao menor e maior valor de espessura equivalente de água encontrado na RHANO no mês em questão (maio ou dezembro) e o valor médio considera a média aritmética do somatório dos valores na região para o referido mês.

Nota-se nos gráficos expostos da figura 9 (a) e (b) uma certa regularidade de 2002 a 2008, um leve acréscimo de 2009 a 2011 e desde então um declínio. 
Comparando-se com o gráfico da figura 6, nota-se equivalência no que diz respeito a relação precipitação versus disponibilidade hídrica, isto é, onde a variação de precipitação apresentou magnitude maior, com picos bem altos no período de 2002 a 2011, a espessura equivalente de água manteve-se regular, já a partir de 2011 quando a variação de precipitação teve menor amplitude, com média baixa. Logo este fato tem reflexo na disponibilidade hídrica e fica exemplificado nos gráficos das figuras 9 (a) e (b), apresentando uma queda a partir de 2011.
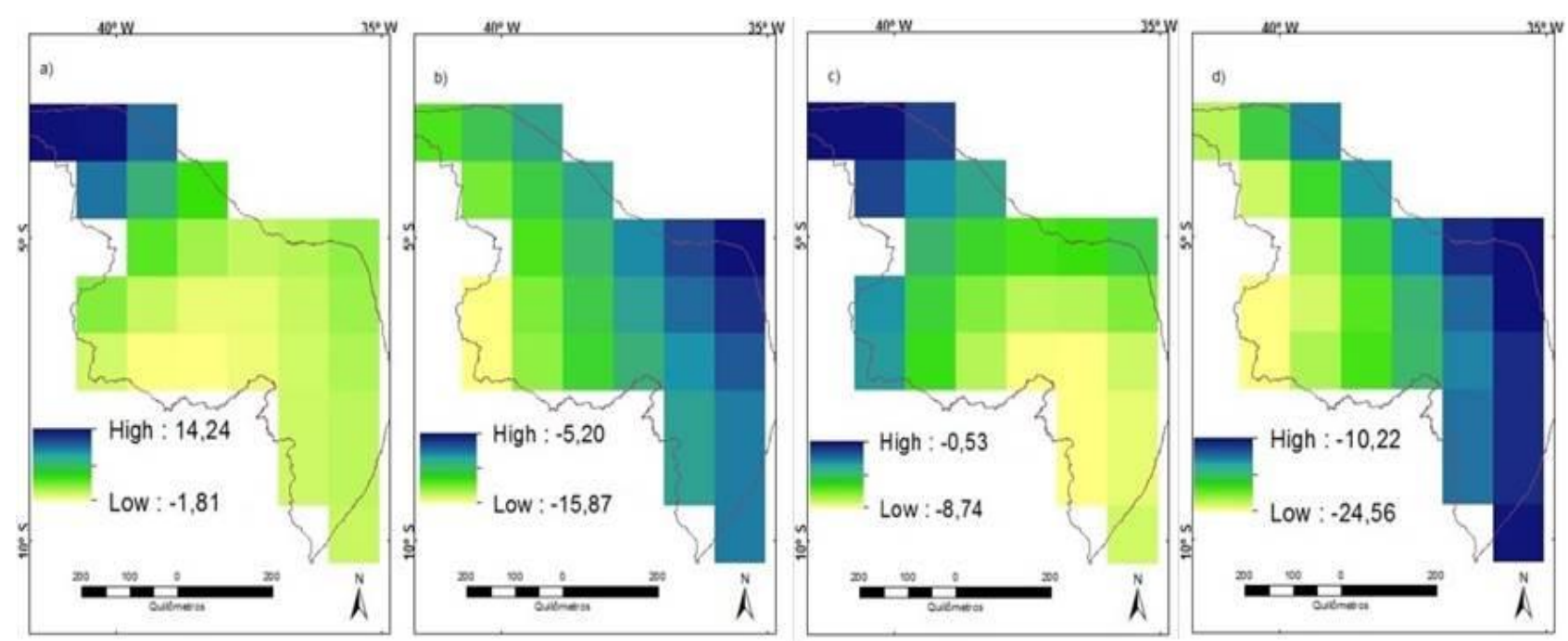

Figura 10 - Espessura equivalente de água (cm) na Região do Atlântico Nordeste Oriental: a) maio de 2002, b) novembro de 2002, c) abril de 2015 e d) dezembro de 2015.

Espessuras equivalente de água obtida pelo GRACE na Região do Atlântico Nordeste Oriental considerando os respectivos meses e anos: maio/2002, novembro/2002; abril/2015e dezembro/2015.

A figura 10 representa a situação de espessura equivalente de água na Região Hidrográfica do Atlântico Nordeste Oriental no final do primeiro semestre de 2002 e de 2015. A escala variando de "High" e "low" nos mapas da figura 10 representam o valor máximo e mínimo de espessura equivalente de água encontrado na RHANO no específico mês e ano em questão.

Quando observado no mesmo ano a variação de espessura de água equivalente representa a variação sazonal anual do período de maiores e menores precipitações (comparações "a" com "b" para 2002 e "c" com "d" para 2015). No entanto, na comparação entre o mesmo mês, ou próximos, mas de anos diferentes ("a" com "c" e "b" com "d"), nota-se uma pequena diferença, a qual reflete uma mudança na quantidade de massa de água disponível entre os 13 anos observados. Sendo assim, a figura 11 apresenta os resultados da subtração da imagem matricial de espessura equivalente de água de abril de 2015 menos maio de 2002 e de dezembro de 2015 menos novembro de 2002.

As comparações temporais apresentadas na figura 11 representam que a porção noroeste da Região Hidrográfica do Atlântico Nordeste Oriental está com as maiores diferenças em espessura de água equivalente, o que reflete uma grande variabilidade entre a quantidade de precipitação sazonal na região ao longo do intervalo de 13 anos, 2002-2015, e tem como consequência uma diferenciação na quantidade de reserva de água disponível.

A porção sul apresentou pouca variabilidade, enquanto a central apresenta um nível intermediário.

Contudo, todas apresentaram redução entre 2002 e 2015 no que concerne a disponibilidade hídrica.

A partir dos cálculos estatísticos obtiveramse as médias de perda de espessura equivalente de água por pixel resultando em uma diferença entre os anos extremos considerados de $-8,3 \mathrm{~cm}$ para maio e $-6,7 \mathrm{~cm}$ para novembro. $\mathrm{O}$ sinal negativo indica perda de volume de água, pois em seu cálculo foram subtraídos os dados de 2015 menos 2002.

Sendo assim a variação de massa entre maio de 2002 com relação a abril de 2015 teve como resultado uma perda no volume de água na região na ordem de $23,7 \mathrm{~km}^{3}\left(23710,4 \mathrm{hm}^{3}\right)$ e na comparação de novembro de 2002 com dezembro de 2015 a perda na disponibilidade 
hídrica foi de $19,2 \mathrm{~km}^{3}\left(19246,1 \mathrm{hm}^{3}\right)$. Para se ter uma ideia comparativa o maior açude do Brasil, o Castanhão no estado do Ceará, possui uma capacidade $6700 \mathrm{hm}^{3}$ (ANA, 2009) e abastece a região da Grande Fortaleza e o Complexo Portuário do Pecém.

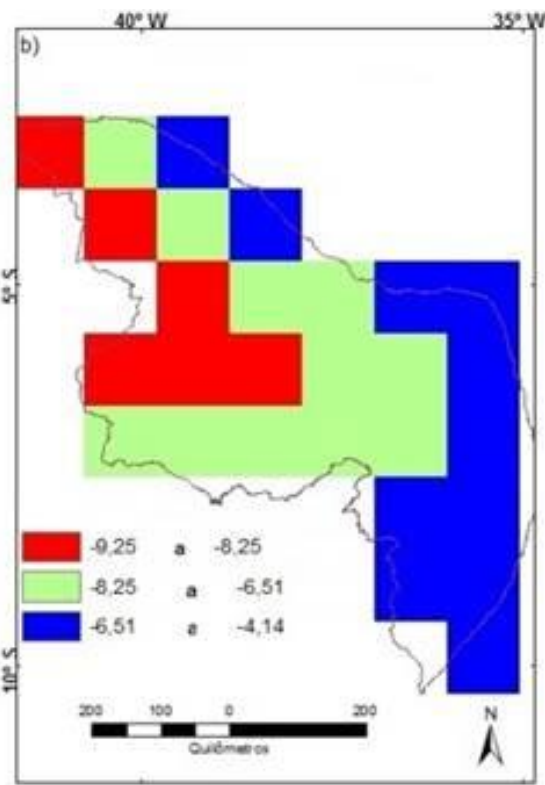

Figura 11 - Diferença de espessura equivalente de água na Região do Atlântico Nordeste Oriental: a) abril de 2015 por maio de 2002 e b) dezembro de 2015 por novembro de 2002. O vermelho, verde e azul mostram, respectivamente, onde houve maiores, intermediárias e menores diferenças entre 2015 e 2002.

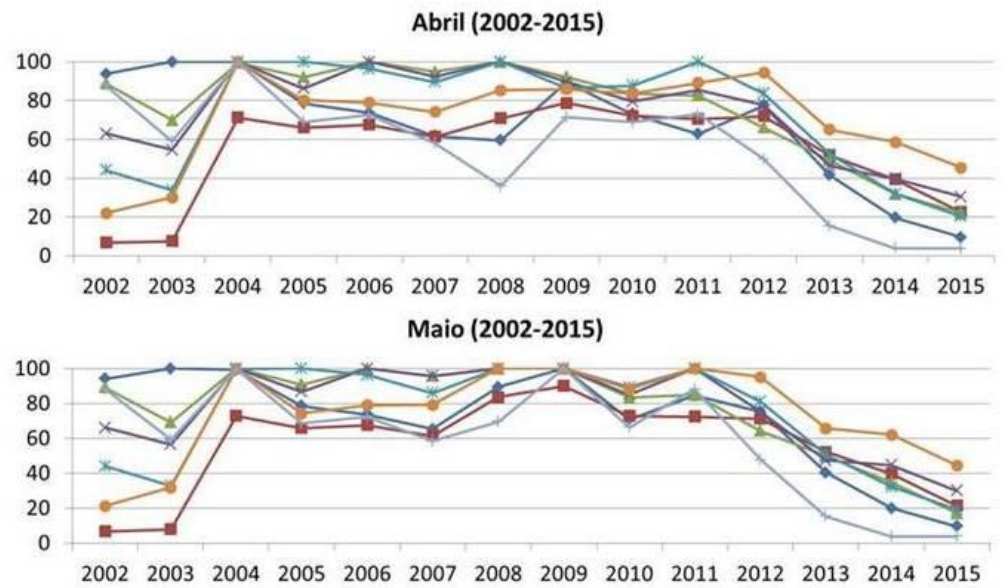

20022003200420052006200720082009201020112012201320142015

Novembro (2002-2015)

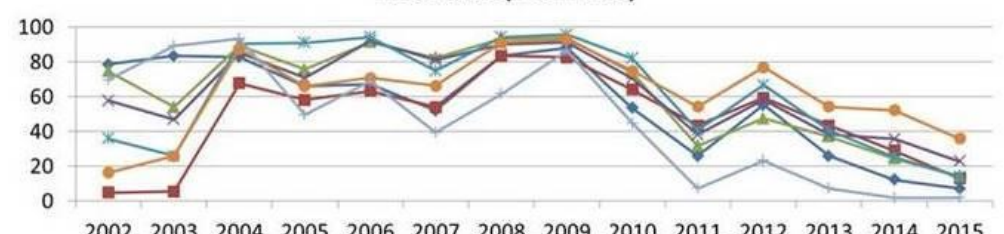

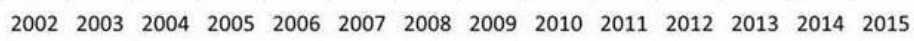

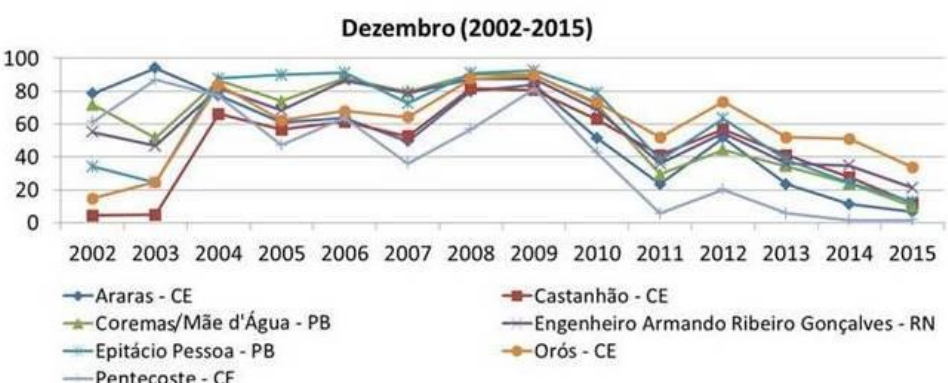

Figura 12 - Situação dos reservatórios na Região Hidrográfica do Atlântico Nordeste Oriental de 2002 a 2015 para os meses de abril, maio, novembro e dezembro 
Série temporal (2002 a 2015) dos principais reservatórios na na Região do Atlântico Nordeste Oriental obtidos da Agência Nacional de Águas (ANA).

$\mathrm{Na}$ figura 12 são apresentados os gráficos de situação dos reservatórios apontados na figura 3 para os meses de abril, maio, novembro e dezembro de 2002 a 2015. É possível detectar três situações distintas de forma geral no que se refere ao comportamento temporal considerando inicialmente os meses de abril e maio: (i) a primeira tendência para o intervalo de 2002 a 2004 onde os reservatórios apresentam uma forte recuperação do percentual de suas reservas hídricas, (ii) de 2004 a 2012 os mesmos mantiveram suas reservas de certa forma equilibradas e (iii) de 2012 a 2015 período onde todos entram em declínio. Já para os gráficos de novembro e dezembro: (i) de 2004 a 2007 mantiveram-se com suas reservas equilibradas, (ii) de 2008 e 2009 observa-se aumento dos níveis e (iii) desde 2010 a 2015 estão em queda. Essa comparação corrobora com as análises anteriores representadas pelas informações obtidas com satélites artificias a respeito da precipitação (TRMM) e espessura equivalente de água (GRACE).

\section{CONCLUSÕES}

A partir das séries temporais apresentadas foi possível analisar as variações de armazenamento d'água para o período de abril de 2002 a dezembro de 2015 bem como identificar os momentos sazonais de maior e menor disponibilidade hídrica na Região Hidrográfica Atlântico Nordeste Oriental. Considerando os extremos da série temporal analisada (2002 e 2015) foi possível calcular a diferença de disponibilidade hídrica nos finais do primeiro e segundo semestre destes anos. Resultado esse que apontou uma perda na ordem de aproximadamente $23,71 \mathrm{~km}^{3}$ para o final do primeiro semestre anual e em torno de $19,25 \mathrm{~km}^{3}$ para o final do segundo semestre.

As informações provenientes dos satélites da missão GRACE vêm a auxiliar na gestão dos recursos hídricos do Brasil e sua potencialidade e divulgação para estudos hidrológicos devem ser exploradas. Destaca-se que a principal vantagem do uso do GRACE é sua disponibilidade de informações para a sociedade de forma gratuita. Já como desvantagens, têm-se que a série temporal ainda é considerada pequena para estudos hidrológicos de longo prazo, pois sua operação, iniciou-se a partir de 2002. Outra desvantagem se refere a sua resolução espacial, não sendo adequada para detectar pequenos sinais de um reservatório específico, por exemplo.

Existem várias outras formas de complementar e dar continuidade aos estudos hidrológicos de uma bacia ou até mesmo mudando a área alvo para outras regiões hidrográficas e/ou agregando uma área maior que comporte várias regiões hidrográficas adjacentes, mas com regime de precipitações semelhantes. Um enfoque diferenciado que pode ser dado é comparar os cálculos de disponibilidade hídrica feitas a partir dos dados gravimétricos da missão com os relatórios fornecidos pelos órgãos responsáveis sobre os recursos hídricos, que segundo a Infraestrutura Nacional de Dados Espaciais INDE (CINDE-CONCAR, 2010), é do Conselho Nacional de Recursos Hídricos (CNRH) e da Agência Nacional de Águas (ANA).

Ressalta-se, que a Região Hidrográfica Atlântico Nordeste Oriental merece atenção especial dos seus gestores, pois a bacia se encontra (a partir de 2012) em estado de alerta, e vem indicando declínio no provimento de água para a sociedade.

\section{AGRADECIMENTOS}

Gostaríamos de agradecer ao suporte dos seguintes projetos: $\mathrm{PQ} /$ nível $2 / \mathrm{CNPq} \mathrm{n}^{\circ}$ do processo 310412/2015 - 3/PQ e PVE/CAPES n ${ }^{\circ}$ do processo 88881.068057/2014-01.

\section{REFERÊNCIAS}

AGÊNCIA NACIONAL DE ÁGUAS (Brasil). Conjuntura dos recursos hídricos no Brasil 2009. Brasília: ANA, 2009.

AGÊNCIA NACIONAL DE ÁGUAS (Brasil). Conjuntura dos recursos hídricos no Brasil: regiões hidrográficas brasileiras - Edição Especial. Brasília: ANA, 2015.
AWANGE, J.L.; FOROOTAN, E.; KUSCHE, J.; KIEMA, J.B.K.; OMONDI, P.A.; HECK, B.; FLEMING, K.; OHANYA, S.O.; GONCALVES, R.M. Understanding the decline of water storage across the Ramser-Lake Naivasha using satellite-based methods. Advances in Water Resources, v. 60, p. 7-23, 2013. 
CONSELHO NACIONAL DE RECURSOS HÍDRICOS CNRH (Brasil). Resolução no. 32, de 15 de outubro de 2003. Divisão Hidrográfica Nacional. Diário Oficial da União 17 dez, 2003.

CHEN, J.L.; WILSON, C.R.; TAPLEY, B.D.; SCANLON, B.; GUNTNER, A. Long-term groundwater storage change in Victoria, Australia from satellite gravity and in situ observations. Global and Planetary Change, v. 139, p. 56-65, 2016.

GETIRANA, A.C.V. Extreme water deficit in Brazil detected from space. Journal of Hydrometeorology, v. 17, n. 2, p. 591599, 2016.

GUTIÉRREZ, A.P.A.; ENGLE, N.L.; NYS, E.; MOLEJON, C.; MARTINS, E.S. Drought preparedness in Brazil. Weather and Climate Extremes, v. 3, p. 95-106, 2014.

HUFFMAN, G.J.; ADLER, R.F.; RUDOLF, B.; SCHNEIDER U.; KEEHN P.R. Global precipitation estimates based on a technique for combining satellite-based estimates, rain gauge analysis, and NWP model precipitation information. Journal of Climate, v. 8, p. 1284-1295, 1995.

HUFFMAN, G.J.; ADLER, R.F.; BOLVIN D.T.; GU, G.; NELKIN, E.J.; BOWMAN, K.P.; HONG, Y.; STOCKER, E.F.; WOLFF, D.B. The TRMM Multisatellite Precipitation Analysis (TMPA): Quasi-Global, Multiyear, CombinedSensor Precipitation Estimates at Fine Scales. Journal of Hydrometeorology, v. 8, p. 38-55, 2007.

JAXA (Japan Aerospace Exploration Agency). Tropical Rainfall Measuring Mission "TRMM". 2015 Disp. em: http://global.jaxa.jp/projects/sat/trmm/. Acessado em: 08 nov 2016.

KUMMEROW, C.; BARNES W.; KOZY T.; SHIUE, J.; SIMPSON, J. The tropical rainfall measuring mission (TRMM) sensor package. Journal of atmospheric and oceanic technology, v. 15, n. 3, p. 809-817, 1998.

LANDERER, F.W. \& SWENSON, S.C. Accuracy of scaled GRACE terrestrial water storage estimates. Water Resources Research, v. 48, p. 1-11, 2012.

MENESES, P.R. \& ALMEIDA, T.D. Introdução ao Processamento de Imagens de Sensoriamento Remoto. Brasília, 2012, 266p. Universidade de Brasília, Brasília.

MINISTÉRIO DO PLANEJAMENTO, ORÇAMENTO E GESTÃO - Comissão Nacional de Cartografia (CONCAR) Comitê de Planejamento da Infraestrutura Nacional de Dados Espaciais (CINDE-CONCAR). Plano de Ação para Implantação da Infraestrutura Nacional de Dados Espaciais. Brasilia. Versão homologada em Janeiro/2010.

MOLODTSOVA, T.; MOLODTSOV, S.; KIRILENKO, A.; ZHANG, X.; VANLOOY, J. Evaluating flood potential with GRACE in the United States. Natural Hazards and Earth System Sciences, v. 16, p. 1011-1018, 2016.
MONTECINO, H.C.; STAUB, G.; FERREIRA, V.G.; PARRA, L.B. Monitoring Groundwater Storage in Northern Chile Based on Satellite Observations and Data Simulation. Boletim de Ciências Geodésicas, v. 22, n. 1, p. 1-15, 2016.

NDEHEDEHE, C.; AWANGE, J.; AGUTU, N.; KUHN, M.; HECK, B. Understanding changes in terrestrial water storage over West Africa between 2002 and 2014. Advances in Water Resources, v. 88, p. 211-230, 2016.

SUN, T.; FERREIRA, V.G.; HE, X.; ANDAM-AKRFUL, S.A. Water. Availability of São Francisco River Basin Based on a Space-Borne Geodetic Sensor. Water, v. 8, n. 5, p. 1-20, 2016.

SWENSON, S.C. \& WAHR, J. Post-processing removal of correlated errors in GRACE data. Geophysical Research Letters, v. 33, p. 1-4, 2006.

SWENSON, S.C. GRACE monthly land water mass grids NETCDF RELEASE 5.0. Ver. 5.0. PO.DAAC, CA, USA. 2012. Disp. em: http://www.jpl.nasa.gov/. Acessado em: $21 \mathrm{mar} 2016$.

TAPLEY, B.D. \& REIGBER, C. The GRACE Mission: Status and Future Plans. Earth and Space Science News. American Geophysical Union, v. 82, n. 47, 2001.

TAPLEY, B.D.; BETTADPUR, S.; WATKINS, M.; REIGBER, C. The Gravity Recovery and Climate Experiment: Mission overview and early results. Geophysical Research Letters, v. 31, p. 1-4, 2004.

TUCCI, C.E.M. Hidrologia: ciência e aplicação. 2.ed. Porto Alegre: ABRH/Editora da UFRGS, $4^{a}$ ed., 944p., 2007.

WAHR, J.; MOLENARR, M.; BRYAN, F. Time variability of the Earth's gravity field: Hydrological and oceanic effects and their possible detection using GRACE. Journal of Geophysical Research, v. 103 (B12), p. 30205-30229, 1998.

WATKINS, M. \& BETTADPUR, S. The GRACE Mission: The Challenges of Using Micron-Level Satellite-to-Satellite Ranging to Measure The Earth's Gravity Field. In: INTERNATIONAL SYMPOSIUM ON SPACE DYNAMICS, 2000, Biarritz, France. Proceedings...Biarritz, p. $1-8,2000$.

XAVIER, L.N.R.; ROTUNNO FILHO, O.C.; CAZENAVE, A.; ARAUJO, A.A.M. Reflexões Sobre Modelagem Hidrológica com Suporte de Dados da Missão Espacial GRACE na Bacia Amazônica. In: SIMPÓSIO BRASILEIRO DE RECURSOS HÍDRICOS, XX, 2013, Bento Gonçalves. Anais... Bento Gonçalves: ABRH, v. 1, p. 1-8.

Submetido em 28 de novembro de 2016 Aceito em 14 de junho de 2018 